\section{GW23-e2581 INTERFERENCE WITH AKT SIGNALING IN DYSLIPIDEMIA DIMINISHES MYOCARDIAL INFARCTION AND PROMOTES SURVIVAL BY INHIBITING OXIDATIVE STRESS}

doi:10.1136/heartjnl-2012-302920a.154

${ }^{1}$ Lining Ma, ${ }^{2}$ Bethany A Kerr, ${ }^{2}$ Xiaoxia Z West, ${ }^{2}$ Nikolay L Malinin, ${ }^{2}$ Malory E Weber, ${ }^{3}$ Liang Ding, ${ }^{4}$ Payaningal R Somanath, ${ }^{5}$ Marc S Penn, ${ }^{2}$ Eugene A Podrez, ${ }^{2}$ Tatiana V Byzova, 'Lining Ma. ${ }^{1}$ Department of Molecular Cardiology, Joseph J. Jacobs Center for Thrombosis and Vascular Biology, Lerner Research Institute, Cleveland Clinic, Cleveland, OH, 44195, USACardiovascular Department, Hainan Provincial People's Hospital, Hainan, China; ${ }^{2}$ Department of Molecular Cardiology, Joseph J. Jacobs Center for Thrombosis and Vascular Biology, Lerner Research Institute, Cleveland Clinic, Cleveland, OH, 44195, USA; ${ }^{3}$ Thrombosis and Vascular Biology, Lerner Research Institute, Cleveland Clinic, Cleveland, OH, 44195, USA; ${ }^{4}$ Clinical and Experimental Therapeutics, College of Pharmacy, University of Georgia, Augusta, GA, 30912; ${ }^{5}$ Integrative Medical Sciences, Northeast Ohio Medical University, Rootstown, $\mathrm{OH}, 44272$

Objectives Cardiovascular disease resulting in myocardial infarction (MI) is the leading cause of mortality in developed countries; however, the mechanisms controlling this complex disorder remain elusive. Since Akt activation was shown to be increased in human and mouse atherosclerotic lesions and failing hearts, we hypothesized that Akt signaling might contribute to several aspects of the pathogenesis of cardiovascular disease.

Methods $\mathrm{C} 57 \mathrm{BL} / 6, \mathrm{ApoE}^{-/-}$(C57BL/6 background), and SR$\mathrm{BI}^{+/-} \mathrm{ApoE}^{-/-}$(mixed C57BL/6xS129 background) mice were purchased from Jackson Laboratories (Bar Harbor, ME). SR$\mathrm{BI}^{+/-} \mathrm{ApoE}^{-/-}$mice were backcrossed to C57BL/6 background for 10 generations. Akt1 $1^{-/}$mice were generated as previously described) and backcrossed to the C57BL/6 background for 10 generations. $\mathrm{SR}-\mathrm{BI}^{-/-} \mathrm{ApoE}^{-/-}$(DKO) mice were generated by intercrossing SR$\mathrm{BI}^{+/-} \mathrm{ApoE}^{-/-}$mice. SR-BI-/-ApoE ${ }^{-/-} \mathrm{Akt1}^{-/-}$(TKO) mice were generated by first crossing $\mathrm{Akt} 1^{-/-}$mice with $\mathrm{SR}-\mathrm{BI}^{+/-} \mathrm{ApoE}^{-/-}$mice. Mice were sacrificed at 42 days of age for most experiments. Immunoblotting

Hearts were homogenized in sample buffer. Proteins were detected with anti-Akt1, anti-phospho-Akt, anti-phospho-GSK-3a/ $\beta$ or anti-GSK-3 $\beta$ antibodies. Cardiac function was evaluated weekly using a $14 \mathrm{MHz}$ linear array transducer interfaced with a Sequoia C256 (Acuson) when mice were between 4 and 7 wk of age. Once clear views of the long axis and the short axis were achieved, $M$ mode analysis was performed to measure ejection fraction (EF) and fraction of shortening (FS). Mice were sacrificed at 6 weeks of age when cardiac dysfunction was just becoming apparent to assess the role of Akt1 deletion in cardiac pathogenesis. Hearts were immersion-fixed in buffered $10 \%$ formalin. Hearts were cut at the mid level transversely, as reported previously, and embedded in paraffin. Serial sections $(8 \mu \mathrm{m})$ were cut and stained with H\&E or Masson's trichrome. To quantify fibrosis, five images were taken of four sections per Masson's trichrome stained heart 
on an Olympus BX51 microscope. Cardiac fibrosis was quantified by measuring the total stained area the total area of the heart using Image Pro.

Atherosclerotic Lesion Measurements

Atherosclerotic lesions were quantified by en face aortic coverage measured by computer-assisted planimetry. Aortae were cut open longitudinally, stained with Oil red $\mathrm{O}$, and digitally scanned. Lesion area was assessed using Adobe Photoshop software.

En Face Immunostaining

VCAM-1 expression was assessed in endothelial cells, as demonstrated by CD31 staining, in the aortic arch and descending thoracic aorta by en face staining, followed by laser-scanning confocal microscopy (Leica TCS-SP) as previously described.

In situ TUNEL Staining

Hearts were embedded in OCT freezing medium and sectioned at $7 \mu \mathrm{m}$. Sections were then fixed in 4\% PFA and analyzed for TUNEL staining using the In Situ Cell Death Detection Kit, TMR Red (Roche) according to the manufacturer's protocol.

In situ detection of superoxide

Dihydroethidium (DHE, Invitrogen) staining for superoxide was carried out as previously described.

Results Akt1 deletion under dyslipidemia alleviates cardiac dysfunction?TKO EF37.67\% \pm 6.60 vs DKO $21.94 \% \pm 3.78$ ? TKO FS $39.25 \% \pm 3.23$ vs DKO $16.22 \% \pm 3.09$ ?, diminishes MI size (TKO $3.78 \% \pm 0.864$ vs DKO $22.02 \% \pm 5.926$ ), and, most importantly, prolongs lifespan DKO $44.23 \pm 7.43$ daysvs TKO $51.43 \pm 6.29$ days?. TKO mice exhibit reduced atherosclerosis. While dyslipidemia was equal, ROS generation and consequent oxidized lipid accumulation was dramatically reduced in TKO. Simultaneously, Akt1 deletion diminished CD36 expression, the main oxidized lipid receptor responsible for foam cell formation.

Conclusions Interference with Akt activation improves survival during dyslipidemia by reducing oxidative stress and oxidized lipid responses thus providing a protective effect. Normalization or prevention of Akt overactivation during atherogenesis might be beneficial for the treatment of atherosclerosis and heart failure. 